26 with no antihistamines. No significant difference was observed in sex ratio, age, family history of febrile seizures or epilepsy, cause of fever, and proportion of simple and complex febrile seizures between antihistamine and non-antihistamine groups. In the antihistamine group, time from fever detection to seizure onset (simple and complex separately or together) was significantly shorter $(\mathrm{P}<0.001)$, and seizure duration was significantly longer $(\mathrm{P}<0.05)$. Seizure types and EEG abnormalities were similar in the two groups. Hypothalamic neuronal histamine depletion induced by antihistamines may lower febrile seizure threshold and increase seizure susceptibility.(Takano T, Sakaue Y, Sokoda T, et al. Seizure susceptibility due to antihistamines in febrile seizures. Pediatr Neurol April 2010;42:277-279). (Respond: Dr Takano, Dept Pediatrics, Shiga University of Medical Science, Otsu, 520-2192, Japan. E-mail: tmyktkn@belle.shigamed.ac.jp).

COMMENT. This clinical study supports the significance of many prior laboratory experiments that demonstrate an increase in seizure susceptibility following antihistamine administration. The febrile seizure threshold temperature was lowered and severity of experimental seizures exacerbated in animals following diphenhydramine administration (Millichap JG et al. Neurology 1960;10:575). West syndrome has been associated with antihistamines in Japan (Yasuhara A et al, 1998; Yamashita Y et al, 2004). Patients with seizures should be cautioned to avoid antihistamine medications when possible.

\title{
MELATONIN AND INTRACTABLE EPILEPSY
}

The relation of serum melatonin levels to sleep patterns and effects on seizure severity were evaluated in 23 children with intractable epilepsy compared to 14 with controlled seizures, in a study at Ain Shams University, Cairo, Egypt. The children with intractable epilepsy had significantly higher scores in sleep walking, teeth grinding, and sleep apneas, compared to those with controlled epilepsy. Diurnal/nocturnal melatonin levels were not significantly different in patients with controlled seizures (10.79/28.7 $\mathrm{pg} / \mathrm{ml}$ ) and uncontrolled seizures $(21.9 / 15.2 \mathrm{pg} / \mathrm{ml})$. Diurnal levels in patients with controlled seizures were significantly lower than levels in a healthy control group (10.79 vs 25.95). Patients with intractable epilepsy received $1.5 \mathrm{mg}$ regular release melatonin 30 min before bedtime for 3 months, in addition to their anticonvulsants that were not changed. An initial dose of $3 \mathrm{mg}$ melatonin was changed to $1.5 \mathrm{mg}$ because 2 patients had increased seizures. With oral melatonin, sleep disorders, including enuresis, sleep walking, sleep apnea, and Epworth sleepiness scores improved in patients with intractable epilepsy. Reduction in seizure severity was significant, but reduction in seizure frequency in $20(87 \%)$ patients was not significant. Seizures increased in frequency in $3(13 \%)$. EEGs became normal in 3 patients. Two patients had headache, skin rash, and abdominal pain. (Elkhayat HA, Hassanein SM, Tomoum HY, et al. Melatonin and sleep-related problems in children with intractable epilepsy. Pediatr Neurol April 2010;42:249-254). (Respond: Dr Tomoum, 10 El-Nagah Street, El-Nozha, Cairo 11361, Egypt. E-mail: tomoumh@yahoo.com). 
COMMENT. In this uncontrolled study of melatonin $(1.5 \mathrm{mg})$ in children with refractory epilepsy, sleep continuity and some sleep-related disorders were significantly improved. Seizure severity, but not frequency, also showed significant improvement.

\section{INTERICTAL EEG SPIKES IDENTIFY SEIZURE FOCUS}

A computerized spike detector was used to measure and localize interictal epileptiform discharges (IEDs) over prolonged, representative segments of recordings in 19 children with intractable, mostly extratemporal lobe epilepsy. Approximately 8 hours of IEEG, randomly selected 30-min segments of continuous interictal IEEG per patient, were analyzed over all intracranial electrode contacts. Spike frequency was averaged over the 16-time segments, and electrodes with the highest mean spike frequency were within the seizure-onset region in 11 of 19 patients. Large statistical samples of interictal activity were required for improved localization. Low-voltage fast EEG at seizure onset was the only factor predicting IED localization to the seizure-onset region. Automated IED detection over multiple samples of IEEG may be of value in planning epilepsy surgery for intractable epilepsy in children. (Marsh ED, Peltzer B, Brown MW, et al. Interictal EEG spikes identify the region of electrographic seizure onset in some, but not all, pediatric epilepsy patients. Epilepsia 2010;51(4):592-601). (Respond: Eric D Marsh MD PhD, Division of Child Neurology, Children's Hospital of Philadelphia, 3400 Civic Center Blvd, Philadelphia, PA 19104. E-mail: marshe@email.chop.edu).

COMMENT. Ictal recording is the mainstay of localizing epileptic foci for surgical resection. The inconsistent correlation with interictal spikes in previous reports may be related to the relatively short periods of observation. Further research is recommended to determine which patients may benefit from this technique.

\section{VASCULAR DISORDERS}

\section{MINOR ATHLETIC TRAUMA AND ISCHEMIC STROKE}

Three cases of arterial ischemic stroke (AIS) following sports-related neck trauma are reported from Johns Hopkins University School of Medicine, Baltimore, MD. Case I. A 10-year-old boy developed a left hemiparesis within a few minutes of colliding with another soccer player and sustaining a hit on his right head and neck. MRI revealed a right basal ganglia stroke. MRA showed no evidence of arterial dissection, and echocardiogram was normal. Treatment with heparin (UFH) followed by aspirin resulted in complete recovery. He was heterozygous for factor V mutation and MTHFR C677T polymorphism. Case 2. A 12-year-old boy sustained right neck and shoulder trauma during a lacrosse game. Later that night, he developed tingling of his right face and headache, and the next morning he awoke with vomiting, right-sided weakness, followed by nystagmus, truncal ataxia, and dysmetria of the right upper and lower limbs. MRI confirmed stroke in the right posterior pontomedullary junction. Traumatic vertebral artery dissection was suspected, and heparin was administered. Digital subtraction angiography (DSA) performed $12 \mathrm{~h}$ after admission showed no dissection, and heparin 